\title{
Bill, Ralf (2016): Grundlagen der Geo-Informationssysteme. 6. völlig neu bearbeitete und erweiterte Auflage
}

\author{
Berlin/Offenbach: Wichmann-Verlag. 855 S., Illustrationen, Diagramme, Karten
}

\author{
Gotthard Meinel ${ }^{1}$ iD
}

Eingegangen: 21. Juni 2016 / Angenommen: 30. Juni 2016 / Online publiziert: 14. Juli 2016

(C) Springer-Verlag Berlin Heidelberg 2016

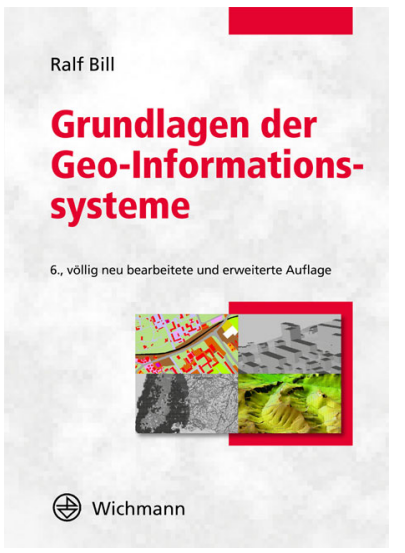

Mit Ralf Bills Standardwerk „Grundlagen der Geo-Informationssysteme“, seit nunmehr 25 Jahren auf dem Markt, wurde inzwischen eine ganze Generation von Geographen, Geodäten, Geoinformatikern und Planern ausgebildet. Er schafft es wie kein anderer, die keineswegs trivialen Grundlagen und komplexen Zusammenhänge der verschiedenen, die Geoinformatik speisenden Technologien - von den mathematischen Grundlagen bis zu Datenbank- und Präsentationstechnologien - höchst verständlich darzustellen.

Nachdem die 5. Auflage aus dem Jahr 2010 vergriffen war, verlangte der Markt eine Neuauflage. Diese 6. Auflage wurde von Ralf Bill genutzt, neue Entwicklungen und Anwendungen in dem sich weiterhin sehr dynamisch entwickelnden Umfeld der Geo-Informationssysteme (GIS) zu beschreiben. Das Buch ist in seiner Neuauflage auf 855 Sei-

\footnotetext{
Dr.-Ing. Gotthard Meinel

g.meinel@ioer.de

1 Leibniz-Institut für ökologische Raumentwicklung, Weberplatz 1, 01217 Dresden, Deutschland
}

ten angewachsen, die das Grundwissen in sehr konzentrierter Form darlegen. Für Wissensvertiefungen, die häufig in der Praxis nötig werden, bietet Ralf Bill ein 30 Seiten umfassendes Literaturverzeichnis. Wichtigste Ergänzungen sind die integrative Behandlung des Themas ,Zeit“ als vierte Dimension von Geo-Informationssystemen, neue Datenbank- und Geovisualisierungstechniken sowie die Entwicklung von Geodateninfrastrukturen, die moderne Verwaltungen längst durchdringen und tragen.

Raumplanung und Geoinformatik sind letztlich einander symbiotisch verbunden, denn eine gute, sprich nachhaltige Raumplanung kann nur auf der Grundlage exakter Raumentwicklungsanalysen und fundierten Prognosen, modellgestützten Szenarien bzw. Projektionen erfolgen. Raumund Umweltanalysen sind aber das Ergebnis komplexer GIS-Anwendungen. Auch die Geoinformatik profitiert von der Raumplanung, stellen doch deren komplexe Fragestellungen sie immer wieder vor neue Herausforderungen. Auch wenn junge Raumplaner und Geographen inzwischen dank fundierter GIS-Ausbildungen Kenntnisse in den Berufsalltag mitbringen, werden diese häufig noch nicht in anspruchsvollen raumanalytischen Arbeiten angewandt. Hier eröffnet sich ein großes Entwicklungspotenzial, zumal die Verfügbarkeit von Geobasis- und Geofachdaten, Rasterdaten der Statistik bis hin zu nutzergenerierten Daten immer besser wird.

Das Buch ist noch reicher illustriert als frühere Ausgaben, was die Verständlichkeit des Inhalts sehr unterstützt. Am Ende eines jeden Kapitels werden Aufgaben gestellt, die dem Leser eine unmittelbare Anwendung des neuen Wissens ermöglichen - eine in Zeiten zunehmenden Selbststudiums hervorragende Möglichkeit der Selbsttestung. Am Ende des Buches finden sich dann die Lösungen. Jedes Kapitel endet mit einer Zusammenfassung, was für eilige Leser ein großer Gewinn ist. Das sehr umfassende Abkürzungs- 
verzeichnis ist bei der Fülle neuer Abkürzungen unverzichtbar.

Das Buch umfasst neun Kapitel. Eine Einführung in GIS und seine wichtigsten Grundlagen bietet das erste Kapitel, welches Begriffsbestimmungen, Entwicklungsgeschichte und Grundmodelle enthält sowie auf GIS-Organisationen, -ausbildungsaspekte und wichtige GIS-Lehrbücher und -Zeitschriften verweist. Die Informationen aus diesem Kapitel sind unverzichtbar für das Verständnis von GIS und sollten zum Grundwissen auch jedes Raumplaners gehören.

Das zweite Kapitel „GIS-Hard- und Software“, welches GIS-Architekturen und -Produkte behandelt, wurde aktualisiert und es wurde nun auch auf Cloud-Technologien und Web-App-Grundlagen eingegangen. Dies mag ein Kapitel sein, welches ein Planer eher nur durchblättert, da es doch etwas tiefer in die Informatik einsteigt.

Im dritten Kapitel „Raum und Zeit in GIS“ werden Raumbezug, Koordinatenreferenzsysteme, geodätische Transformationen und die Zeit als vierte Dimension in GIS behandelt. Nachdem in Geoinformationssystemen der Raum früher nur statisch modelliert wurde, ist eine dynamische Betrachtung häufig unabdingbar geworden. Hiervon wird der Planer profitieren, werden doch Entwicklungsanalysen dadurch erleichtert bzw. überhaupt erst ermöglicht. So können perspektivisch, beispielsweise auf der Basis von ALKIS (Amtliches Liegenschaftskatasterinformationssystem), auch Entwicklungsanalysen des Gebäudebestands erstellt werden, da Anfang und Ende eines jeden Gebäudes modellmäßig erfasst werden.

Das Kapitel „Interoperabilität und offene GIS-Welten“ gibt einen Überblick zu diesem sich rasant entwickelnden komplexen Themenfeld. Neben Modellsprachen und weltweiter Normungsaktivität werden hier auch die europäischen INSPIRE-Bemühungen zum Aufbau einer Geodateninfrastruktur, Bundes-, Landes-, regionale und lokale GDI-Aktivität (Geodateninfrastruktur) und im Kapitel „Digital Earth“ auch Copernicus, Geosensornetzwerke, 3DStadt-, Bauwerks- und Landschaftsmodelle behandelt. Die Raumplanung profitiert von diesen Entwicklungen enorm, denn neue Daten werden europaweit weitestgehend harmonisiert und Dienste-orientiert verfügbar sein.

Das Kapitel „Erfassung raumbezogener Daten“ wurde um die Themen Umweltmessnetze und nutzergestützte Datenerhebungen erweitert, die mit Projekten wie OpenStreetMap (OSM) längst ihr großes Nutzungspotenzial nachgewiesen haben. In diesem Kapitel wird auch auf das Thema Metainformationen und Datenqualität eingegangen. Diese sind für GIS-Anwender von größter Bedeutung, sind doch
Metadaten noch heute oft nur unvollständig vorliegend. Hier haben selbst große Datenanbieter, allen voran Vermessungsbehörden, die als staatliche Einrichtung eine besondere Verantwortung tragen, immer noch Hausaufgaben zu machen.

Im nachfolgenden Kapitel „Raumbezogene Datenverwaltung" wird auf geometrische, topologische und thematische Modellierung sowie logische und physikalische Datenmodelle eingegangen. Das so wichtige Thema Datenbanksysteme wird allerdings nur sehr knapp behandelt, hier ist für eine Vertiefung ganz sicher Sekundärliteratur hinzuzuziehen, auf die in großer Zahl verwiesen wird.

Die Kapitel „Raumbezogene Datenanalysemethoden“, „Präsentation raumbezogener Daten“ und „Anwendungen von GIS“ sind für den Raumplaner sicher die interessantesten, da hier die unmittelbaren Grundlagen empirischer räumlicher Analysearbeit dargestellt werden: Verschneidung, Nachbarschafts- und Netzwerkanalyse, Zonengenerierung, Interpolation, Klassifikation und Geostatistik. Auch neuere Analysemethoden, wie das „Big Data“ und „Spatial Data Mining“ oder Kostenoberflächen in GIS, werden thematisiert.

In „Präsentation raumbezogener Daten“ wird unter anderem auf die sich rasant entwickelnden 3D-Visualisierungstechniken, erweiterte Realitäten mit den Zielrichtungen „Virtual Reality“, „Augmented Reality“ und „Mixed Reality“, die physische Welten mit Rechnermodellen vermischen, eingegangen. Hier wird auch die Bedeutung interoperabler GIS-Umgebungen hervorgehoben, die mit definierten OGC-Webservices (Open Geospatial Consortium) Realität wurden.

Im Kapitel 9 „Anwendungen von GIS“ wird überblicksartig über Land-, Raum-, Umwelt- und Netzinformationsund spezielle Fachinformationssysteme informiert. Hier wird auch detailliert auf das $\mathrm{AAA}^{\circledR}$-Modell eingegangen, auf dessen Grundlage inzwischen digitale Geobasisdaten flächendeckend für Deutschland bereitgestellt werden, die längst Grundlage der meisten Planungsprozesse sind.

Das Buch ist nicht nur Studierenden wärmstens zu empfehlen, sondern auch Praktikern, insbesondere der Bereiche Raum- und Umweltplanung. Im Grunde genommen ist die Lektüre aber für alle ein Gewinn, die sich für Methoden zur Lösung raumbezogener Probleme und die Hintergründe raumbezogener Anwendungen interessieren. Letztere durchdringen längst unseren Alltag, denn wer fährt noch ohne „Navi“ in fremde Städte oder verzichtet auf ortsbezogene Dienste, hilfreich nicht nur bei der Geschäfts-, Restaurant- und Wohnungssuche oder auf der Urlaubsreise? 